\title{
LES CONNAISSANCES EN SOINS PALLIATIFS DES INFIRMIÈRES QUI TRAVAILLENT EN EMS AUPRÈS DE PERSONNES ÂGÉES NOTAMMENT ATTEINTES DE DÉMENCE
}

\author{
Elisabete Iori, Diane Morin
}

Médecine \& Hygiène | « Revue internationale de soins palliatifs 》

2015/3 Vol. 30 | pages 109 à 117

ISSN 1664-1531

Article disponible en ligne à l'adresse :

http://www.cairn.info/revue-infokara-2015-3-page-109.htm

\section{!Pour citer cet article :}

Elisabete Iori, Diane Morin, « Les connaissances en soins palliatifs des infirmières qui travaillent en EMS auprès de personnes âgées notamment atteintes de démence ", Revue internationale de soins palliatifs 2015/3 (Vol. 30), p. 109-117.

DOI 10.3917/inka.153.0109

Distribution électronique Cairn.info pour Médecine \& Hygiène.

(C) Médecine \& Hygiène. Tous droits réservés pour tous pays.

La reproduction ou représentation de cet article, notamment par photocopie, n'est autorisée que dans les limites des conditions générales d'utilisation du site ou, le cas échéant, des conditions générales de la licence souscrite par votre établissement. Toute autre reproduction ou représentation, en tout ou partie, sous quelque forme et de quelque manière que ce soit, est interdite sauf accord préalable et écrit de l'éditeur, en dehors des cas prévus par la législation en vigueur en France. Il est précisé que son stockage dans une base de données est également interdit. 

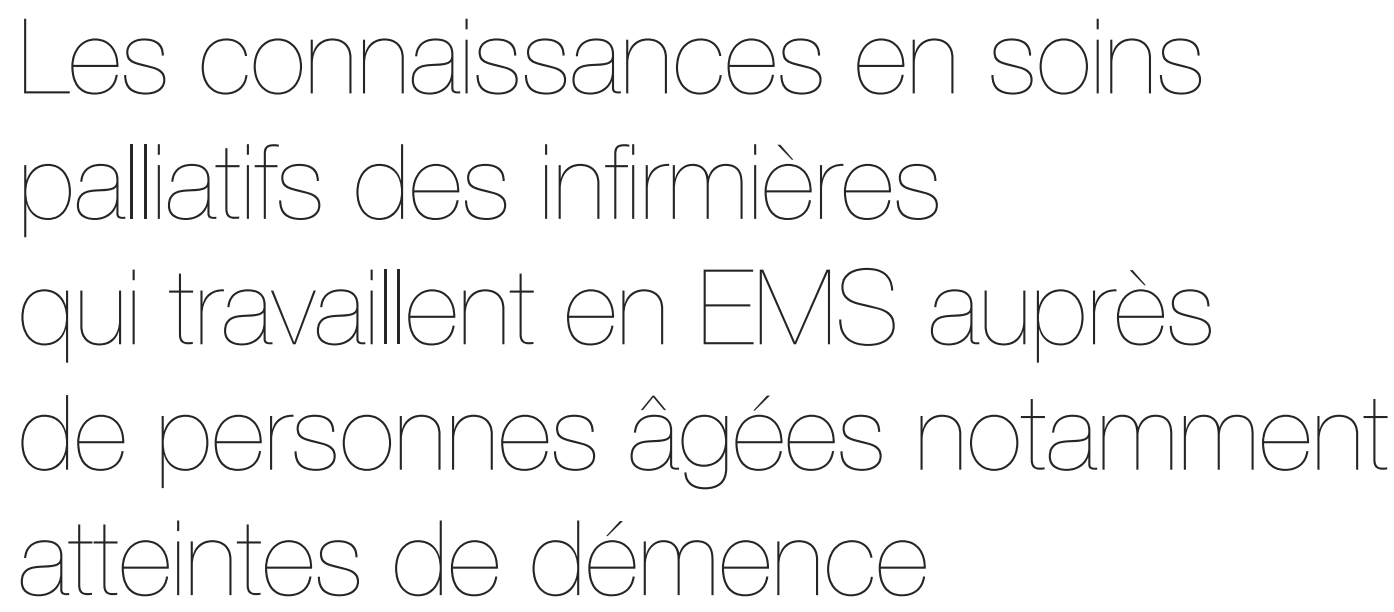

\section{Elisabete lori}

Inf., MSc, Infirmière clinicienne spécialisée, Centre Neuchâtelois de Psychiatrie (CNP), Neuchâtel

\section{Diane Morin}

Infirmière PhD. Directrice de l'Institut universitaire de formation et recherche en soins, (IUFRS), Lausanne

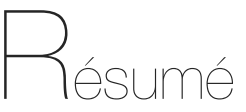

Connaissances en soins palliatifs des infirmières qui travaillent en EMS auprès de personnes âgées, notamment atteintes de démence - Le vieillissement de la population et l'augmentation du nombre de personnes atteintes de démence sont des enjeux pour les soins de fin de vie en EMS (Établissements Médico-Sociaux). Les connaissances des infirmières dans ce domaine méritent d'être examinées.

Notre but a été d'évaluer leurs connaissances à l'aide du Palliative Care Knowledge Test. Des analyses descriptives et des corrélations ont été effectuées.

Le score moyen a été de $61,5 \%$. Près de $16 \%$ des participants ont $\leq 50 \%$ de bonnes réponses alors que seulement $5 \%$ en ont eu $\geq 80 \%$. Aucun participant n'a eu plus que $84 \%$. Des corrélations faibles mais significatives apparaissent entre un score plus élevé et l'âge, le nombre d'années depuis le diplôme et le nombre d'années d'expérience en EMS. Les connaissances des soignants en matière de soins palliatifs en EMS sont faibles mais elles augmentent avec l'âge et l'expérience professionnelle. II serait pertinent de promouvoir de la formation continue en soins palliatifs et le soutien des soignants de manière à assurer une prise en charge des personnes vieillissantes et démentes en fin de vie.<smiles>Br[Bi](Br)c1ccccc1</smiles>

Palliative care knowledge of nurses working in residential nursing homes for the elderly, particularly in respect to those suffering from dementia - The ageing population and the increase in the number of people suffering from dementia present a challenge for endof-life care in residential nursing homes (EMS). The nurses' knowledge in this area is worth examining. Our aim was to evaluate their knowledge using the Palliative Care Knowledge Test. Descriptive analyses and correlations were made.

The average score was $61.5 \%$. Close to $16 \%$ of participants had $\leq 50 \%$ correct answers whereas only $5 \%$ had $\geq 80 \%$. No participant scored more than $84 \%$. Weak but significant correlations appeared between higher scores and age, the number of years since graduation and the number of years worked in EMS.

The nursing staff's knowledge of palliative care in EMS is poor but improves with age and professional experience. It would be appropriate to promote vocational training in palliative care and support for the carers in order to ensure the care of ageing people with dementia at the end of life. 


\section{Introduction}

\section{Contexte}

L

e vieillissement de la population dans les der-

nières années a mis en exergue de nouveaux

défis pour les soignants qui sont préoccupés par le maintien d'une qualité de vie optimale pour les personnes âgées, notamment en fin de vie. Aussi, avec ce changement démographique, l'incidence et la prévalence de maladies chroniques et dégénératives tendent à augmenter et la fin de vie avec démence devient de plus en plus commune. En Suisse, le nombre de personnes de 65 ans et plus est de 1,3 million en 2010 (soit 17\% de la population) et de celles-ci environ 84000 (environ 6\%) vivent dans un établissement médico-social (EMS) parce qu'elles présentent des besoins élevés d'aide et de soins que leur réseau sociofamilial ne peut offrir [1]. Selon l'Association Alzheimer Suisse [2], en 2010, environ 107000 personnes sont atteintes de la maladie d'Alzheimer ou d'une autre forme de démence et pas moins de 25000 nouveaux cas sont diagnostiqués chaque année. Ces chiffres seraient en augmentation selon l'Office Fédéral de la Statistique (OFS). Le rapport de 2008/09 de l'OFS nous indique que $39 \%$ des personnes vivant en EMS seraient atteintes de démence [3]. En 2009, la démence a été classée comme faisant partie des trois principales causes de décès, avec les maladies cardiovasculaires et le cancer [4].

\section{Soins palliatifs à la personne démente}

Actuellement le concept des soins palliatifs ne se limite plus à la prise en charge des pathologies oncologiques et n'est plus exclusif aux services spécialisés hospitaliers. Toutes les pathologies, notamment les démences, peuvent nécessiter le recours à ces soins et de plus en plus ils s'étendent aux soins à domicile et aux EMS. Dans cette optique, les soins palliatifs doivent être connus, la philosophie qui les supporte doit être comprise par tous les soignants afin de favoriser le développement et l'application de meilleures pratiques cliniques possibles pour toutes les personnes en fin de vie. Selon certains auteurs, l'approche générale des soins palliatifs est appropriée à la prise en charge de la personne démente dans l'optique d'une amélioration de la qualité des soins de fin de vie [5]. Les besoins en soins palliatifs de la personne démente ne sont pas toujours reconnus puisque la démence présente un cours qui est souvent très long et que les patients déments atteints d'une défaillance cognitive et communicationnelle poseront des défis particuliers dans la prise en charge, ainsi que des questions éthiques liées à l'autonomie et à la prise de décision [6, 7, 8]. Malgré que les infirmières et les autres membres du personnel soignant dans les EMS soient de plus en plus formés et qualifiés, il y a des études qui montrent que la formation continue sur la mort et les soins à la personne âgée n'est pas toujours privilégiée dans ces milieux $[9,10,11]$. Chang et al. [12] identifient d'ailleurs un manque de connaissances des soignants en soins palliatifs. Ils considèrent qu'il s'agit d'un challenge dans la prise en charge de la personne démente. La formation et l'éducation sont donc considérées essentielles afin que les soins palliatifs à la personne démente deviennent une réalité [13]. En Suisse, et plus spécifiquement dans les cantons romands, aucune étude n'a porté sur l'évaluation des connaissances en soins palliatifs des infirmières travaillant en EMS auprès de personnes atteintes de démence.

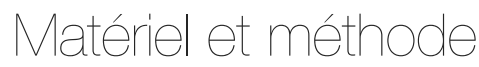

\section{Dispositif de recherche}

Cette recherche est de nature quantitative descriptive transversale et inclut un volet corrélationnel exploratoire. Elle a pour but d'évaluer les connaissances en soins palliatifs chez des infirmières travaillant avec des personnes atteintes de démence, en EMS.

\section{Population, méthode d'échantillonnage et collecte de données}

La population cible de cette étude est composée de toutes les infirmières travaillant en EMS dans un canton suisse romand. Ainsi, les critères d'inclusion étaient de travailler en EMS dans le canton en question et de comprendre le français. Puisque toutes les infirmières des EMS participants ont été invitées à participer, l'échantillon a été construit par convenance. La collecte de données s'est déroulée entre le mois de novembre 2012 et le mois de mars 2013. La taille d'échantillon a été calculée afin de pouvoir mesurer des coefficients de corrélations de 0,60 entre la variable dépendante, soit le score total à l'échelle des connaissances et certaines variables indépendantes telles que l'expérience. L'échantillon requis pour observer une corrélation de cette envergure a été estimé à 55.

\section{Variables, instruments, et propriétés psychométriques}

Les caractéristiques des infirmières ont été recueillies par le moyen d'un questionnaire qui comprend 
différentes variables sociodémographiques dont: le niveau de formation, l'expérience professionnelle, l'expérience dans le domaine des soins palliatifs de même que la formation dans le domaine des soins palliatifs. Les connaissances en soins palliatifs ont été évaluées par le moyen d'un questionnaire intitulé «Palliative Care Knowledge Test» (PCKT) [14]. Préalablement, ce questionnaire a été traduit, validé et adapté en français suisse selon la méthode de Wild [15]. Pour chacun des 37 énoncés retenus dans le questionnaire adapté au français suisse, trois modalités de réponse sont possibles: Vrai, Faux, Je ne sais pas. Le score se compose des scores de réponses correctes par énoncé, par dimension et pour l'ensemble de l'échelle. Plus le score est élevé, plus le niveau de connaissance est considéré comme bon. Les variables étudiées par ce questionnaire sont la philosophie des soins, la douleur, la dyspnée, les troubles psychiatriques, les troubles gastro-intestinaux et la communication.

\section{Analyse statistique}

L'analyse des données a été effectuée à l'aide du logiciel Stata $12^{\circledR}$. La base de données a été créée dans le logiciel Excel. Dans un premier temps, des analyses univariées ont été faites de manière à avoir une vision globale des caractéristiques des variables. Ensuite, des analyses bivariées ont permis d'évaluer la relation entre les scores du PCKT et des données sociodémographiques.

\section{Résultats}

\section{Données sociodémographiques des participants}

Les participants à l'étude sont essentiellement du sexe féminin (87,65\%) d'âge moyen de 43 ans. La personne la plus jeune qui a répondu au questionnaire a 23 ans et la plus âgée en a 63. En ce qui concerne les diplômes, $39,5 \%$ des répondants ont des diplômes suisses, contre une majorité de 49,4\% de diplômes français et 11,1\% de diplômes obtenus dans d'autres pays dont le Portugal $(\mathrm{n}=5)$, la Belgique $(\mathrm{n}=1)$, la Serbie $(\mathrm{n}=1)$, la Slovaquie $(\mathrm{n}=1)$, ou le Québec $(\mathrm{n}=1)$. Pour les diplômes suisses $(\mathrm{n}=32)$, $21,9 \%$ sont des infirmières-assistantes, $43,8 \%$ sont des diplômes niveau I et II, 18,6\% sont des diplômes de niveau ES et 15,6\% des diplômes de niveau HES. Dans l'échantillon de l'analyse du «nombre d'années d'expérience » $(\mathrm{n}=80)$, la moyenne a été de 19,5 , avec un minimum de 1 et un maximum de 40 ans. Le «nombre d'années d'expérience en EMS » $(\mathrm{n}=81)$ est en moyenne de 11,3 avec une valeur minimum de
0 et maximum de 28 ans. La majorité des infirmières ont un pourcentage de travail qui varie entre $60 \%$ et $80 \%$ avec une moyenne de taux d'activité $73,3 \%$. Seulement 25,9\% des participants ont obtenu des certifications de formation continue suite à leur formation de base. Il s'agit de formations post-grade $(n=10)$ en management, soins palliatifs et personne âgée, puériculture, gérontologie, psychiatrie et psychogériatrie, ou encore des certificats d'études avancés (CAS) $(n=10)$ en santé mentale, soins palliatifs, praticien formateur, gestion d'équipe et gestion de projets et encore des études de Master $(n=1)$. Au vu de la petite taille des sous-échantillons, et de l'impossibilité de faire une analyse d'association avec le score total du questionnaire, deux catégories ont été créées (diplômes de formation continue - «oui» ou «non») pour pouvoir réaliser cette analyse. En moyenne, les infirmières ont pris en charge 2,9 patients qui ont eu besoin de soins palliatifs dans les derniers 3 mois. Les valeurs du nombre de patients qui ont eu besoin de soins palliatifs varient entre 0 et 20. Dans les 12 derniers mois, seulement 12 infirmières (14.8\%) ont eu des heures de formation en soins palliatifs. Ces heures de formation varient entre 0 et 180 heures avec une moyenne de 3,8 et une médiane de 0 . De fait, ces formations étaient généralement courtes. À la question concernant la présence d'un référent en soins palliatifs dans l'institution, dans $82,7 \%$ des cas la réponse a été négative.

\section{Scores du PCKT}

\section{Score des réponses correctes par énoncé}

La fréquence et le pourcentage des réponses correctes par énoncé sont présentés dans le tableau 1. Le pourcentage de réponses correctes par énoncé a oscillé entre $18,5 \%$ et $98,8 \%$. Les deux énoncés avec les scores les plus élevés sont: "Ce qui est important pour la qualité de vie varie d'un individu à l'autre.» et "Les compétences et les techniques en communication peuvent s'apprendre.» (98,8\%). La réponse avec un score moins élevé est à l'énoncé "Les niveaux de saturation en oxygène influencent la dyspnée» (18,5\% de réponses exactes), suivi par «L'utilisation d'opiacés à long terme souvent induit une accoutumance. » (27,2\%) et «L'information incertaine ne devrait pas être donnée aux patients ou à la famille parce que cela pourrait causer de l'anxiété additionnelle. » $(28,4 \%)$. Des 37 énoncés, presque la moitié soit $13(35,1 \%)$ ont eu un score de réponses correctes inférieur à 50\%. Seulement 5 énoncés ont eu un score correct plus haut que 90\%. 
Tableau 1: Scores pour l'ensemble, par dimension et par énoncé de l'échelle PCKT.

Score moyen pour l'ensemble

\section{Dimensions et énoncés \\ Dimensions et énoncés}

$61,49 \%$ (Écart type 0,10$)$
$\%$ des réponses correctes

\section{Dimension: Philosophie de soins}

1. Les soins palliatifs devraient être dispensés seulement aux patients pour lesquels aucun traitement curatif n'est disponible.

2. Les soins palliatifs ne devraient pas être dispensés en même temps que les traitements anticancéreux.

3. Ce qui est important pour la qualité de vie varie d'un individu à l'autre.

4. Les patients qui reçoivent des soins palliatifs doivent accepter la mort.

Dimension: Douleur

5. Quand un patient cancéreux a de la douleur, les opiacés devraient être introduits en premier.

6. Un des buts de la gestion de la douleur est de pouvoir bénéficier d'une bonne nuit de sommeil.

7. Une fois que les opiacés sont administrés, les médicaments anti-inflammatoires non stéroïdiens ne devraient pas être utilisés.

8. L'utilisation régulière d'analgésiques est efficace pour la gestion de la douleur liée au cancer.

9. Les antidépresseurs et anticonvulsivants parfois aident à soulager la douleur liée au cancer.

10. Même si des douleurs aigües surviennent lorsque des opiacés sont utilisés de manière régulière, la prochaine dose ne devrait pas être donnée plus tôt que prévu.

11. L'utilisation d'opiacés à long terme souvent induit une accoutumance.

12. L'utilisation de laxatifs est efficace pour prévenir la constipation induite par les opiacés.

13. L'augmentation du dosage des opiacés devrait être limitée parce qu'une dépression respiratoire peut survenir comme effet secondaire.

14. L'utilisation des opiacés n'influence pas la durée de survie du patient.

Dimension: Dyspnée

15. La dyspnée peut être diminuée par l'usage de la morphine.

16. Quand les opiacés sont pris de manière régulière et encore avec le but de diminuer la dyspnée, la dépression respiratoire est facilement induite.

17. Les niveaux de saturation en oxygène influencent la dyspnée

18. L'évaluation de la dyspnée devrait se baser sur la perception subjective des patients.

19. Les médicaments anticholinergiques ou la scopolamine $\left(B \cup S C O P A N^{\circledR}\right)$ sont efficaces pour atténuer les sécrétions bronchiques chez les patients en fin de vie.

20. La seule façon efficace d'atténuer les sécrétions bronchiques chez les patients en fin de vie est l'aspiration manuelle.

Dimension: Troubles psychiatriques

21. Durant les derniers jours de vie, la non-correction du déséquilibre électrolytique et la déshydratation peuvent augmenter le confort du patient.

Fréquence

des réponses

correctes

91,05 (Écart type 0,16)

\begin{tabular}{l|r}
72 & 88,89
\end{tabular}

\begin{tabular}{l|l} 
& \\
\hline 74 & 91,36
\end{tabular}

\begin{tabular}{|l|c|}
\hline 74 & 91,36 \\
\hline 80 & 98,77 \\
\hline 69 & 85,19 \\
\hline 63,83 (Écart type 0,16) \\
\hline
\end{tabular}

\begin{tabular}{|l|l|}
\hline 57 & 70,37 \\
\hline
\end{tabular}

\begin{tabular}{|c|c|}
\hline 58 & 71,60 \\
\hline 47 & 58,02 \\
\hline
\end{tabular}




\section{Score des réponses correctes par dimension}

La dimension Philosophie a été celle avec une plus haute moyenne de réponses correctes $(91,1 \%)$ suivie par Communication $(72,4 \%)$ et Douleur $(63,8 \%)$. La dimension avec une moyenne de réponses correctes plus basse a été Troubles gastro-intestinaux $(50,9 \%)$, bien que très proche des moyennes présentées par les dimensions Dyspnée (54,3\%) et Troubles Psychiatriques (54,3\%). Le Tableau 1 expose les résul- tats pour le score total obtenu par les infirmières dans le Palliative Care Knowledge Test. En moyenne, les infirmières ont eu $61,5 \%$ de réponses correctes. Pour ce qui est de la valeur minimale ou maximale, le score le plus bas a été de 40,5\% et le plus élevé de $83,8 \%$. Seulement quatre infirmières $(4,9 \%)$ ont eu un score supérieur à $80 \%$ mais 13 infirmières (16,0\%) ont eu un score total inférieur à 50\%.

Tableau 1: Scores pour l'ensemble, par dimension et par énoncé de l'échelle PCKT (suite).

\begin{tabular}{|c|c|c|}
\hline Dimensions et énoncés & $\begin{array}{l}\text { Fréquence } \\
\text { des réponses } \\
\text { correctes }\end{array}$ & $\begin{array}{l}\% \\
\text { des réponses } \\
\text { correctes }\end{array}$ \\
\hline \multicolumn{3}{|l|}{ Dimension: Troubles psychiatriques (suite) } \\
\hline $\begin{array}{l}\text { 22. Les benzodiazépines devraient être efficaces pour contrôler le délirium/état } \\
\text { confusionnel. }\end{array}$ & 36 & 44,44 \\
\hline $\begin{array}{l}\text { 23. Certains patients en fin de vie auront besoin de sédation continue pour atténuer } \\
\text { leur souffrance. }\end{array}$ & 72 & 88,89 \\
\hline 24. La morphine est souvent une cause de délirium chez les patients en phase terminale. & 39 & 48,15 \\
\hline $\begin{array}{l}\text { 25. Le délirium apparaît fréquemment chez les patients qui sont sujets à une fai- } \\
\text { blesse mentale (angoisse, anxiété). }\end{array}$ & 35 & 43,21 \\
\hline Dimension: Troubles gastro-intestinaux & \multicolumn{2}{|c|}{ 50,89 (Écart type 0,17) } \\
\hline $\begin{array}{l}\text { 26. Une sonde gastrique devrait être insérée pour diminuer les symptômes causés } \\
\text { par une obstruction du tractus digestif. }\end{array}$ & 43 & 53,09 \\
\hline $\begin{array}{l}\text { 27. En phase terminale d'un cancer, une ingestion hypercalorique est plus nécessaire } \\
\text { qu'aux stades précédents. }\end{array}$ & 39 & 48,15 \\
\hline $\begin{array}{l}\text { 28. Une diminution d'apport hydrique par voie orale liée au cancer peut seulement } \\
\text { être traitée par une perfusion. }\end{array}$ & 42 & 51,85 \\
\hline $\begin{array}{l}\text { 29. Même si l'apport hydrique par voie orale n'est pas possible à cause d'une obs- } \\
\text { truction du tractus digestif, la nutrition hypercalorique parentérale améliore sou- } \\
\text { vent la qualité de vie si l'état général du patient est bon. }\end{array}$ & 51 & 62,96 \\
\hline $\begin{array}{l}\text { 30. II n'y a pas d'autre voie qu'un cathéter veineux central pour les patients inca- } \\
\text { pables de garder une voie veineuse périphérique. }\end{array}$ & 39 & 48,15 \\
\hline $\begin{array}{l}\text { 31. Lorsque les patients reçoivent une perfusion de } 1000 \mathrm{~mL} \text { ou plus par jour, l'épan- } \\
\text { chement pleural et l'ascite ont tendance à augmenter. }\end{array}$ & 43 & 53,09 \\
\hline 32. Les stéroïdes devraient améliorer l'appétit chez les patients avec un cancer avancé. & 27 & 33,33 \\
\hline $\begin{array}{l}\text { 33. Quand les patients avec un iléus utilisent de la métoclopramide (PASPERTIN }{ }^{\circledR} \text {, } \\
\text { PRIMPERAN }^{\circledR} \text { ), les douleurs de l'estomac et les vomissements peuvent se péjorer. }\end{array}$ & 23 & 28,40 \\
\hline $\begin{array}{l}\text { 34. La perfusion intraveineuse ne sera pas efficace pour atténuer la sécheresse buccale } \\
\text { chez les patients en fin de vie. }\end{array}$ & 64 & 79,01 \\
\hline Dimension: Communication & \multicolumn{2}{|c|}{ 72,43 (Écart type 0,17) } \\
\hline 35. Les compétences et les techniques en communication peuvent s'apprendre. & 80 & 98,77 \\
\hline $\begin{array}{l}\text { 36. L'information requise par les patients et familles ainsi que le degré avec lequel } \\
\text { ces derniers prennent part aux décisions peuvent changer en fonction de l'évolution } \\
\text { de la maladie et de la durée de celle-ci. }\end{array}$ & 73 & 90,12 \\
\hline $\begin{array}{l}\text { 37. L'information incertaine ne devrait pas être donnée aux patients ou à la famille } \\
\text { parce que cela pourrait causer de l'anxiété additionnelle. }\end{array}$ & 23 & 28,40 \\
\hline
\end{tabular}




\section{Analyses de corrélation}

Dans l'analyse des variables continues (âge, nombre d'années de diplôme, nombre d'années d'expérience en EMS et pourcentage de travail), les tests d'hypothèse ont montré que ces variables sont faiblement corrélées au score total obtenu dans le PCKT. On peut voir que malgré des corrélations faibles, celles-ci sont significatives pour l'âge $(r=0,3013 ; p=0,007)$, les années de diplôme $(r=0.36 ; p=0,001)$ et les années d'expérience en EMS $(r=0,2553 ; p=0,0214)$. Cela signifie que plus l'âge, les années depuis le diplôme ou les années d'expérience en EMS sont élevées, meilleures sont les connaissances. Pour toutes les variables catégorielles étudiées à un seuil de probabilité à $p>0.05$, aucune différence significative n'a été identifiée.

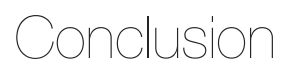

Cette étude a évalué les connaissances en soins palliatifs des infirmières travaillant auprès de personnes atteintes de démence en EMS. Le score moyen total du questionnaire PCKT a été d'environ 61\% de réponses correctes. Un très bas pourcentage des participants $(<5 \%)$ ont eu un score plus élevé que $80 \%$ et environ $15 \%$ des participants ont eu un score total inférieur à 50\% de réponses correctes.

Dans les énoncés avec le score le plus bas, il y a quelques surprises. Environ $81 \%$ des infirmières sont persuadées que le niveau de saturation en oxygène a un lien direct avec la dyspnée et à peu près $37 \%$ des infirmières ne sont pas au clair avec la possibilité de l'utilisation de la morphine pour le soulagement de la douleur. Encore plus étonnant, environ 73\% des infirmières pensent que l'utilisation d'opioïdes pour le soulagement de la douleur induit souvent une accoutumance et environ $42 \%$ croient que l'utilisation de ces médicaments a une influence directe sur la durée de survie du patient. Ces peurs infondées illustrent un manque de connaissances de base sur les pratiques exemplaires en matière d'utilisation des opioïdes dans la gestion de la douleur [8].

D’une manière générale le score total du questionnaire n'est pas très élevé et pour les troubles gastrointestinaux, la dyspnée et les troubles psychiatriques, la moyenne de réponses correctes est seulement légèrement plus élevée que $50 \%$ ce qui laisse à réfléchir que le niveau de connaissances peut être amélioré dans un très grand pourcentage des cas. Vu que cette étude a été pionnière au niveau de la Suisse Romande, il n'y a pas de comparaison possible avec d'autres études. Malgré cela, dans les dernières années, plu- sieurs autres études ont conclu à un manque de connaissances des infirmières concernant les soins palliatifs et ciblent des besoins spécifiques de formation dans ce domaine $[9,10,11,12,13,14,16,17,18,19]$. En 2009, l'Office Fédéral de la Santé Publique [20] avait conclu que l'offre au niveau des soins palliatifs manquait de coordination et qu'elle variait selon les cantons. Selon cette évaluation, l'acquisition de compétences en soins palliatifs dans les formations de base, universitaire, post-grade et continue devrait se faire d'une manière plus systématique. Ces constatations expliquent probablement une partie du fait que dans cette étude, peu d'infirmières ont une formation spécialisée en soins palliatifs. De plus, plusieurs auteurs ont identifié la formation continue des soignants en soins palliatifs comme un point central dans le maintien de la qualité des soins apportés aux patients et aux familles [7, 21, 22, 23, 24, 25, 26, $27,28,29]$

Selon la théorie de l'intervention en soins infirmiers de Sidani et Braden [30], les caractéristiques spécifiques des infirmières ont un effet sur l'intervention et ainsi sur les résultats obtenus. Or, les connaissances en soins palliatifs sont reconnues par plusieurs auteurs comme faisant partie de ces caractéristiques et comme étant un des prérequis dans l'offre de soins de qualité $[14,16,17,18,19]$.

Cette étude a présenté différentes limitations. D'abord, l'accessibilité à 42 EMS (19 établissements seulement ont accepté de participer) a été difficile, puis le faible nombre de participants reste une des limites malgré le fait que nous ayons obtenu le nombre suffisant pour procéder aux corrélations prévues. Aussi, le questionnaire PCKT utilisé pour mesurer les connaissances des infirmières a lui aussi été limitatif en certains points. D'abord, trois des 40 énoncés initiaux ont dû être éliminés du questionnaire suisse parce que les énoncés concernaient d'autres pays ou encore des médicaments non commercialisés en Suisse. Puis, le questionnaire n'a pas été conçu spécifiquement pour mesurer les connaissances en soins palliatifs à la population démente, car il est axé sur les maladies cancéreuses. Malgré cela, il a été adapté dans la mesure des connaissances générales en soins palliatifs des infirmières expertes ayant participé à sa validation pour cette étude. Il serait intéressant de créer une échelle d'évaluation des connaissances en soins palliatifs concernant spécifiquement les soins à une population démente, car ceci implique des connaissances supplémentaires liées à la maladie.

Les analyses de corrélation ont été limitées en certains points en raison de la petite taille des souséchantillons. Ceci s'est avéré exact par exemple pour 
les questions de la formation continue en soins palliatifs. Il serait important de vérifier cette relation entre le niveau de connaissances et la formation en soins palliatifs à travers d'autres études.

Finalement, malgré les avancements et l'augmentation des connaissances en soins palliatifs des soignants travaillant dans des établissements pour personnes âgées, il y a encore des améliorations à faire en soins palliatifs pour assurer une prise en charge efficace des patients avec maladie avancée non maligne, notamment la démence. La solution

\section{Bibliographie}

1. Office Fédéral de la Statistique. Santé des personnes âgées vivantes en établissement médico-social. Enquête sur la santé des personnes âgées dans les institutions 2008/09. Neuchâtel, 2012.

2. Association Alzheimer Suisse. 107000 personnes atteintes d'Alzheimer vivent en Suisse, 2010. Repéré à www.alz.ch

3. Office Fédéral de la Statistique. État de santé des personnes âgées dans les institutions 2008/09. Neuchâtel. Communiqué de presse $N^{\circ} 0351-$ 1005-00. 2010.

4. Office Fédéral de la Statistique. Statistique des institutions médicosociales 2011 - Tableau standard. Résultats définitifs. Neuchâtel, 2013.

5. Meier DE, Lim B, Carlson MD. Raising the standard: palliative care in nursing homes. Health Affairs, 2010, 29(1), 136-140.

6. Gillick M. Doing the right thing: a geriatrician's perspective on medical care for person with advanced dementia. Journal of Law, Medecine \& Ethics, 51-56, 2012

7. Henderson J, Kydd A. Training the trainers: palliative care for people with dementia. Journal of Dementia Care, 20(1), 20-23, 2012.

8. Faull C, de Caestecker S, Nicholson A, Black F. Handbook of Palliative Care. Third edition. New Jersey: Wiley-Blackwell, 2012.

9. Stevens J. McFarlane J, Strirling K. Ageing and dying. In: Kellehear A. (Ed.). Death and dying in Australia. Melbourne: Oxford University Press, 177-189, 2001.

10. Moss M, Braunschweig H, Rubinstein R. Terminal care for nursing home residents with dementia (ethics). Alzheimer's Care Quarterly, 3(3), 233249, 2002.

11. Volicer L. Commentary on «Terminal care for nursing home residents with dementia», by Moss et al. Alzheimer's Care Quarterly, 3(3), 233-249, 2002

12. Chang E, Hancock K, Harrison K, Daly J, Johnson A, Easterbrook S, Noel M, Luhr-Taylor M, Davidson PM. Palliative care for end-stage dementia: a discussion of the implication for education of health care professionals. Nurse Education Today, 25, 326-332, 2005.

13. Jordan A. Extending palliative care to patients with dementia. British Journal of Hospital Medicine, 7(1), 31-35, 2010.

14. Nakazawa Y, Miyashita M, Morita T, Umeda M, Oyagi, Y.Ogasawara T. The palliative care knowledge test: reability and validity of an instrument to mesure palliative care knowledge among health professionals. Palliative Medicine, 23(8), 754-766, 2009.

15. Wild D, Grove A, Martin M, Eremenco S, McElroy S, Verjee-Lorenz A, Erikson P. Principles of Good Practice for the Translation and Cultural Adaptation Process for Patient-Reported Outcomes (PRO) Measures: Report of the ISPOR Task Force for Translation and Cultural Adaptation. Value in Health, 8(2), 94-104, 2005. passe avant tout par la formation continue et le soutien systématique par des experts, pour les soignants qui travaillent dans ces milieux qui sont souvent négligés en comparaison avec les unités hospitalières. Il serait important de faire plus de recherches sur cette thématique des soins palliatifs à la personne démente en EMS. La promotion d'une politique de recherche et d'une pratique basée sur les preuves devrait être mise en valeur dans ces institutions afin d'améliorer la qualité de vie des patients âgés en général et celle des patients atteints de démence en particulier.

16. Abu-Saad Huijer H, Dimassi H, Abboud S. Perspectives on palliative car in Lebanon: knowledge, attitudes and practices of medical and nursing specialties. Palliative and Supportive Care, 7, 339-347, 2009.

17. Waldron M, Hasson F, Kernohan WG, Whittaker E, McClaughlin D. Evaluating education in palliative care with link nurses in nursing homes. British Journal of Nursing, 17(17), 1078-1083, 2008.

18. Thompson S, Bott M, Boyle D, Gajewski B, Tilden VP. A measure of Palliative Care in Nursing Homes. Journal of Pain and Symptom Management, 2012.

19. Mcllfatrick S, Mawhinney A, Gilmour F. Assessing the educational needs of palliative care link nurses. International Journal of Palliative Nursing, 16 (11), 555-559, 2010.

20. Office Fédéral de la Santé Publique. Évaluation des besoins dans le domaine des soins palliatifs. Zurich, 2009.

21. Thomas K. Caring for the dying at home: companions on a journey. Oxford: Radcliffe Medical Press, 2003a.

22. Thomas K. The gold standards Framework in community palliative care. European Journal of Palliative Care, 10(3), 113-115, 2003b.

23. Hall S, Goddard C, Stewart F, Higginson IJ. Implementing a quality improvement program in palliative care in homes: a qualitative study. BMC Geriatrics, 11(31), 1-11, 2011.

24. Reid L, Snowdem A, Kydd, A. An exploration of palliative care provision in scottish care homes. British Journal of Nursing, 21(1), 8-15, 2011.

25. Lester PE, Daroowalla F, Harisingani R, et al. Evaluation of Housestaff Knowledge and Perception of Competence in Palliative Symptom Management. Journal of Palliative Medicine, 14(2), 139-145, 2011.

26. Pelayo M, Cebrian D, Areosa A, Agra Y, Vicente Jl, Buendia F. Effects of online palliative care training on knowledge, attitude and satisfaction of primary care physicians. BCM Family Practice, 12(37), 1-11, 2011.

27. Ryan T, Gardiner C, Bellamy G, Gott M, Ingleton C. Barriers and facilitators to the receipt of palliative care for people with dementia: The views of medical and nursing staff. Palliative Medicine, 26(7), 879-886, 2011.

28. Kuhn DR, Forrest JM. Palliative Care for advanced dementia: a pilot project in 2 nursing homes. American Journal of Alzheimer's Disease and Other Dementias, 27(1), 33-40, 2012.

29. Bloomer MJ, Digby R. Caring for patients dying with dementia: challenges in subacute care in Australia. Journal of Palliative Care, 28(3), 164-168, 2012.

30. Sidani S, Braden CO. Design, Evaluation and Translation of Nursing Interventions. Oxford: Wiley-Blackwell, 2011. 
PCKT - Score des réponses correctes par énoncé.

\begin{tabular}{|c|c|c|c|}
\hline Dimensions et énoncés & $\begin{array}{l}\text { Réponse } \\
\text { correcte } \\
F=\text { faux } \\
\text { V=vrai }\end{array}$ & $\begin{array}{l}\text { Fréquence } \\
\text { des réponses } \\
\text { correctes }\end{array}$ & $\begin{array}{l}\% \\
\text { des réponses } \\
\text { correctes }\end{array}$ \\
\hline Dimension: Philosophie de soins & & \multicolumn{2}{|c|}{ 91,05 (Écart type 0,16) } \\
\hline $\begin{array}{l}\text { 1. Les soins palliatifs devraient être dispensés seulement aux } \\
\text { patients pour lesquels aucun traitement curatif n'est disponible. }\end{array}$ & $\mathrm{F}$ & 72 & 88,89 \\
\hline $\begin{array}{l}\text { 2. Les soins palliatifs ne devraient pas être dispensés en même } \\
\text { temps que les traitements anticancéreux. }\end{array}$ & $\mathrm{F}$ & 74 & 91,36 \\
\hline $\begin{array}{l}\text { 3. Ce qui est important pour la qualité de vie varie d'un individu } \\
\text { à l'autre. }\end{array}$ & V & 80 & 98,77 \\
\hline $\begin{array}{l}\text { 4. Les patients qui reçoivent des soins palliatifs doivent accep- } \\
\text { ter la mort. }\end{array}$ & $\mathrm{F}$ & 69 & 85,19 \\
\hline Dimension: Douleur & & \multicolumn{2}{|c|}{ 63,83 (Écart type 0,16) } \\
\hline $\begin{array}{l}\text { 5. Quand un patient cancéreux a de la douleur, les opiacés } \\
\text { devraient être introduits en premier. }\end{array}$ & $\mathrm{F}$ & 57 & 70,37 \\
\hline $\begin{array}{l}\text { 6. Un des buts de la gestion de la douleur est de pouvoir béné- } \\
\text { ficier d'une bonne nuit de sommeil. }\end{array}$ & V & 58 & 71,60 \\
\hline $\begin{array}{l}\text { 7. Une fois que les opiacés sont administrés, les médicaments } \\
\text { anti-inflammatoires non stéroïdiens ne devraient pas être uti- } \\
\text { lisés. }\end{array}$ & $\mathrm{F}$ & 47 & 58,02 \\
\hline $\begin{array}{l}\text { 8. L'utilisation régulière d'analgésiques est efficace pour la ges- } \\
\text { tion de la douleur liée au cancer. }\end{array}$ & $\mathrm{V}$ & 59 & 72,84 \\
\hline $\begin{array}{l}\text { 9. Les antidépresseurs et anticonvulsivants parfois aident à } \\
\text { soulager la douleur liée au cancer. }\end{array}$ & V & 63 & 77,78 \\
\hline $\begin{array}{l}\text { 10. Même si des douleurs aigües surviennent lorsque des opia- } \\
\text { cés sont utilisés de manière régulière, la prochaine dose ne } \\
\text { devrait pas être donnée plus tôt que prévu. }\end{array}$ & $\mathrm{F}$ & 53 & 65,43 \\
\hline $\begin{array}{l}\text { 11. L'utilisation d'opiacés à long terme souvent induit une accou- } \\
\text { tumance. }\end{array}$ & $\mathrm{F}$ & 22 & 27,16 \\
\hline $\begin{array}{l}\text { 12. L'utilisation de laxatifs est efficace pour prévenir la constipa- } \\
\text { tion induite par les opiacés. }\end{array}$ & V & 76 & 93,83 \\
\hline $\begin{array}{l}\text { 13. L'augmentation du dosage des opiacés devrait être limitée } \\
\text { parce qu'une dépression respiratoire peut survenir comme } \\
\text { effet secondaire. }\end{array}$ & $\mathrm{F}$ & 35 & 43,21 \\
\hline $\begin{array}{l}\text { 14. L'utilisation des opiacés n'influence pas la durée de survie du } \\
\text { patient. }\end{array}$ & V & 47 & 58,02 \\
\hline Dimension: Dyspnée & & \multicolumn{2}{|c|}{ 54,32 (Écart type 0,20) } \\
\hline 15. La dyspnée peut être diminuée par l'usage de la morphine. & $\mathrm{v}$ & 51 & 62,96 \\
\hline $\begin{array}{l}\text { 16. Quand les opiacés sont pris de manière régulière et encore } \\
\text { avec le but de diminuer la dyspnée, la dépression respiratoire } \\
\text { est facilement induite. }\end{array}$ & $\mathrm{F}$ & 26 & 32,10 \\
\hline 17. Les niveaux de saturation en oxygène influencent la dyspnée & $\mathrm{F}$ & 15 & 18,52 \\
\hline $\begin{array}{l}\text { 18. L'évaluation de la dyspnée devrait se baser sur la perception } \\
\text { subjective des patients. }\end{array}$ & $\mathrm{V}$ & 42 & 51,85 \\
\hline $\begin{array}{l}\text { 19. Les médicaments anticholinergiques ou la scopolamine } \\
\left(B \cup S C O P A N{ }^{\circledR}\right) \text { sont efficaces pour atténuer les sécrétions } \\
\text { bronchiques chez les patients en fin de vie. }\end{array}$ & V & 60 & 74,07 \\
\hline $\begin{array}{l}\text { 20. La seule façon efficace d'atténuer les sécrétions bronchiques } \\
\text { chez les patients en fin de vie est l'aspiration manuelle. }\end{array}$ & $\mathrm{F}$ & 70 & 86,42 \\
\hline
\end{tabular}




\begin{tabular}{|c|c|c|c|}
\hline Dimensions et énoncés & $\begin{array}{l}\text { Réponse } \\
\text { correcte } \\
F=\text { faux } \\
V=\text { vrai }\end{array}$ & $\begin{array}{l}\text { Fréquence } \\
\text { des réponses } \\
\text { correctes }\end{array}$ & $\begin{array}{l}\% \\
\text { des réponses } \\
\text { correctes }\end{array}$ \\
\hline Dimension: Troubles psychiatriques & & \multicolumn{2}{|c|}{ 54,32 (Écart type 0,22) } \\
\hline $\begin{array}{l}\text { 21. Durant les derniers jours de vie, la non-correction du désé- } \\
\text { quilibre électrolytique et la déshydratation peuvent augmen- } \\
\text { ter le confort du patient. }\end{array}$ & V & 38 & 46,91 \\
\hline $\begin{array}{l}\text { 22. Les benzodiazépines devraient être efficaces pour contrôler } \\
\text { le délirium/état confusionnel. }\end{array}$ & V & 36 & 44,44 \\
\hline $\begin{array}{l}\text { 23. Certains patients en fin de vie auront besoin de sédation } \\
\text { continue pour atténuer leur souffrance. }\end{array}$ & V & 72 & 88,89 \\
\hline $\begin{array}{l}\text { 24. La morphine est souvent une cause de délirium chez les } \\
\text { patients en phase terminale. }\end{array}$ & $\mathrm{F}$ & 39 & 48,15 \\
\hline $\begin{array}{l}\text { 25. Le délirium apparaît fréquemment chez les patients qui sont } \\
\text { sujets à une faiblesse mentale (angoisse, anxiété). }\end{array}$ & $\mathrm{F}$ & 35 & 43,21 \\
\hline Dimension: Troubles gastro-intestinaux & & \multicolumn{2}{|c|}{ 50,89 (Écart type 0,17) } \\
\hline $\begin{array}{l}\text { 26. Une sonde gastrique devrait être insérée pour diminuer les } \\
\text { symptômes causés par une obstruction du tractus digestif. }\end{array}$ & $\mathrm{F}$ & 43 & 53,09 \\
\hline $\begin{array}{l}\text { 27. En phase terminale d'un cancer, une ingestion hypercalorique } \\
\text { est plus nécessaire qu'aux stades précédents. }\end{array}$ & $\mathrm{F}$ & 39 & 48,15 \\
\hline $\begin{array}{l}\text { 28. Une diminution d'apport hydrique par voie orale liée au cancer } \\
\text { peut seulement être traitée par une perfusion. }\end{array}$ & $\mathrm{F}$ & 42 & 51,85 \\
\hline $\begin{array}{l}\text { 29. Même si l'apport hydrique par voie orale n'est pas possible } \\
\text { à cause d'une obstruction du tractus digestif, la nutrition } \\
\text { hypercalorique parentérale améliore souvent la qualité de vie } \\
\text { si l'état général du patient est bon. }\end{array}$ & V & 51 & 62,96 \\
\hline $\begin{array}{l}\text { 30. II n'y a pas d'autre voie qu'un cathéter veineux central pour les } \\
\text { patients incapables de garder une voie veineuse périphérique. }\end{array}$ & $\mathrm{F}$ & 39 & 48,15 \\
\hline $\begin{array}{l}\text { 31. Lorsque les patients reçoivent une perfusion de } 1000 \mathrm{~mL} \text { ou } \\
\text { plus par jour, l'épanchement pleural et l'ascite ont tendance } \\
\text { à augmenter. }\end{array}$ & V & 43 & 53,09 \\
\hline $\begin{array}{l}\text { 32. Les stéroïdes devraient améliorer l'appétit chez les patients } \\
\text { avec un cancer avancé. }\end{array}$ & V & 27 & 33,33 \\
\hline $\begin{array}{l}\text { 33. Quand les patients avec un iléus utilisent de la métoclopramide } \\
\text { (PASPERTIN }^{\circledR} \text {, PRIMPERAN }{ }^{\circledR} \text { ), les douleurs de l'estomac et } \\
\text { les vomissements peuvent se péjorer. }\end{array}$ & V & 23 & 28,40 \\
\hline $\begin{array}{l}\text { 34. La perfusion intraveineuse ne sera pas efficace pour atténuer la } \\
\text { sécheresse buccale chez les patients en fin de vie. }\end{array}$ & V & 64 & 79,01 \\
\hline Dimension: Communication & & \multicolumn{2}{|c|}{ 72,43 (Écart type 0,17) } \\
\hline $\begin{array}{l}\text { 35. Les compétences et les techniques en communication peuvent } \\
\text { s'apprendre. }\end{array}$ & v & 80 & 98,77 \\
\hline $\begin{array}{l}\text { 36. L'information requise par les patients et familles ainsi que le } \\
\text { degré avec lequel ces derniers prennent part aux décisions } \\
\text { peuvent changer en fonction de l'évolution de la maladie et de } \\
\text { la durée de celle-ci. }\end{array}$ & V & 73 & 90,12 \\
\hline $\begin{array}{l}\text { 37. L'information incertaine ne devrait pas être donnée aux } \\
\text { patients ou à la famille parce que cela pourrait causer de } \\
\text { l'anxiété additionnelle. }\end{array}$ & $\mathrm{F}$ & 23 & 28,40 \\
\hline
\end{tabular}

